\title{
The global Mycobacterium chimaera outbreak
}

\author{
Enrico Tortoli \\ Emerging Bacterial Pathogens Unit, San Raffaele Hospital, Milan, Italy
}

\section{Summary}

Infections due to Mycobacterium chimaera associated with a heater-cooler device used during the open-heart surgery have been reported worldwide. These cases represent the first outbreak caused by a non-tuberculous mycobacterium at a global level. Salient features of the infection are long latency and high mortality. Different aspects of the M. chimaera outbreak, from the first detected cases to the present burden are reviewed in this study. Although the source has been found and thwarted, new cases continue to be detected due to the late manifestation of symptomatology. The treatment remains poorly effective and mortality is around $50 \%$.

\section{Introduction}

Mycobacterium chimaera is a slowly growing species belonging to the Mycobacterium avium complex (MAC). Previously known as ITS1-sequevar MAC-A, it was raised to species rank in 2004 by a polyphasic approach (50). It is actually distinguishable from other MAC members by unique sequences in the 16S rRNA gene and in the internal transcribed spacer (ITS) interposed between the $16 \mathrm{~S}$ and the $23 \mathrm{~S}$ rRNA; conversely, at phenotypic level, it pres-

Correspondence: Enrico Tortoli, Emerging Bacterial Pathogens Unit, San Raffaele Hospital, via Olgettina 60, 20132 Milan, Italy.

E-mail: tortoli.enrico@hsr.it

Key words: Mycobacterium chimaera, outbreak, non-tuberculous mycobacterium.

Conflict of interest: the author declares no potential conflict of interest.

Funding: none.

Received for publication: 17 January 2019.

Revision received: 6 February 2019.

Accepted for publication: 6 February 2019.

(C) Copyright E. Tortoli, 2019

Licensee PAGEPress, Italy

Microbiologia Medica 2019; 34:8037

doi:10.4081/mm.2019.8037

This article is distributed under the terms of the Creative Commons Attribution Noncommercial License (by-nc 4.0) which permits any noncommercial use, distribution, and reproduction in any medium, provided the original author(s) and source are credited. ents a HPLC mycolic acid pattern not found in other MAC members. The name chimaera (the mythological creature made up of parts of three different animals) was assigned due to the apparent mix of genetic features characterizing the species. Out of 12 strains investigated for the species description, seven proved to be responsible for pulmonary disease according to the ATS criteria (1), a finding that led the authors to hypothesize higher pathogenic power in comparison with other MAC members (50). However, this hypothesis has not been confirmed by other studies $(8,39)$.

$M$. chimaera has been frequently isolated from water and is able to form biofilm (55).

Very recently phylogenomic analyses have suggested more appropriate the classification of $M$. chimaera at subspecies level with the name $M$. intracellulare subs. chimaera (47).

Several commercially available DNA probe systems are unsuitable for identification of $M$. chimaera, GenoType Mycobacterium CM (Hain Lifescience, Germany) and AccuProbe M. intracellulare (Hologic, USA) misidentify M. chimaera as M. intracellulare (49). With INNO LiPA Mycobacteria (Fujirebio, Belgium), M. chimaera hybridizes with the probe MIN-2 (48,49); GenoType NTM-DR (Hain Lifescience) identifies M. chimaera as such.

\section{The beginning of the story}

Two fatal cases of $M$. chimaera infection were reported in Zurich (Switzerland) (2), from patients with history of cardiac interventions in 2013. In one case M. chimaera was isolated from both an explanted prosthetic valve and blood culture; in the other, it has grown from bone marrow, blood cultures, urine and tracheal swabs. The strains isolated from the two patients revealed a close relation at genotypic level, while differed from any other strain of $M$. chimaera grown from various sources in the hospital of Zurich. Epidemiological investigations allowed to detect further retrospective cases and led to the implementation of surveillance procedures consisting in culturing for mycobacteria water and air samples collected in operating rooms (36).

\section{The heater-cooler device}

The heater-cooler unit (HCU) is a device used in open-chest surgical interventions to adjust the blood temperature during extracorporeal circulation. HCU has two circuits, the cold one for cardioplegia and the warm one for the blood; the circulating water acts as heat exchanger and does not come into contact with the patient. A fan present in the HCU provides air circulation.

Among the multiple environmental samples collected in the operative rooms at Zurich Hospital (36) some grew in culture $M$. chimaera: from the water of five HCUs and from the air exhausted 
by one HCU. Such findings, in addition to having verified that HCU circuits were not airtight, led to hypothesize the emission from the HCUs of aerosolized $M$. chimaera potentially capable of infecting the surgical area.

Despite the replacement of the HCUs with others, brand new, and the use of frequently changed filtered water, environmental cultures did not steadily convert to negative and, at different times, $M$. chimaera regrew (37). Very soon emerged that HCUs of a specific brand and model (LivaNova, 3T, Germany) were involved and their early contamination, during production in factory, appeared likely $(21,45)$. Starting from September 2014 LivaNova implemented a novel decontamination procedure at the end of the production cycle and provided customers with updated instructions recommending periodic decontaminations of the devices. Only in a few cases $M$. chimaera grew from water collected in HCUs of other brands but none was a source of outbreak.

\section{The spreading of the outbreak}

Following the first report in Switzerland many others were published in Germany (19), Australia (32,34,44),United Kingdom $(13,40)$, Canada $(10,22,29)$, Denmark (46), USA (4$6,23,27,30,35,41)$, Poland $(24)$, Italy $(11,15)$, Cina $(14,56)$ and Spain (3).

\section{The solution of the mystery}

An epidemiological study (53) recently gathered $250 \mathrm{M}$. chimaera strains including 21 clinical isolates associated with HCU use during cardiothoracic surgery and others from patients without history of open-heart interventions, as well as environmental strains from water and air collected at LivaNova factory and in hospitals, both from HCUs and from drinking water. All the strains underwent whole genome sequencing and a phylogenomic tree was built based on single nucleotide polymorphisms. The strains grouped into eight clusters: all those from the $21 \mathrm{HCU}-$ related patients belonged to Group 1, while the remaining clinical strains were dispersed across the tree. A more in-depth cluster analysis split Group 1 in 11 subgroups. Subgroup 1.1 included, along with 20 out of 21 clinical isolates above mentioned, most of the strains isolated from water at LivaNova factory and from air sampled in hospitals, close to working HCUs. The very high clonality of the strains of Subgroup 1.1 proves the evidence of the contamination of HCUs in LivaNova, with only one case (the HCU-associated clinical strain not clustering within Subgroup 1.1) of contamination of the device at hospital level. More recently, also the M. chimaera strains involved in an outbreak at Hong Kong Hospital proven to belong to the Subgroup 1.1 (56).

\section{The disease}

The case definition requires: detection of M. chimaera from invasive specimen, history of open-heart surgery and compatible clinical presentation (40).

Subjects who underwent a surgical intervention requiring cardiopulmonary bypass in the five years prior onset of symptomatology, with valve replacement or reconstruction and aortic vascular graft, are at high risk of infection. The disease is characterized by long latency and mortality may be as high as $60 \%$ (40). Initial symptoms are fever, fatigue and weight loss. Endocarditis and infection, either disseminated or localized at prosthetic vascular graft or sternotomy wound, are the most common manifestations. Other presentations include hepatitis, nephritis, splenomegaly, chorioretinitis, osteomyelitis and bone marrow involvement with cytopenia (17). Development of non-necrotizing granulomatous tissue is frequent.

The optimum treatment is unknown; as for other mycobacterial infections, drugs association is required. In most cases the regimen recommended for pulmonary MAC infection has been adopted: macrolide plus rifampicin and ethambutol (20). The biofilm formation is the most common factor responsible for treatment failure; in such cases a second surgery with replacement of prosthetic material is the only way out (25).

\section{Microbiological diagnosis}

The blood culture is the diagnostic test of choice. It is characterized by good sensitivity but the collection of two or three samples is recommended because of possible intermittent bacteraemia. In case of new surgery, the culture of explanted materials and/or biopsies is mandatory (16). In asymptomatic subjects, the history of surgery with exposition to $3 \mathrm{~T} \mathrm{HCU}$ should warrant blood culture testing.

Some of the commercial methods for identification of mycobacteria are not suited for definition of M. chimaera (26), therefore every identification as $M$. intracellulare or MAC must be checked by sequencing of $16 \mathrm{~S}$ rRNA or ITS.

\section{Environmental sampling}

Mycobacterial cultures should be performed on air and water samples collected in operating rooms while the HCU is working (16). For air sampling proper instrumentations are commercially available which suck selected air volumes $\left(1 / 2 \mathrm{~m}^{3}\right.$ is recommended) and throw particulate, including bacteria, on the surface of an agar plate (selective Middlebrook 7H11 is suggested). The air should be collected close to (30 $\mathrm{cm}$ approx.) the air exhaust of the operating HCU. Water samples should be collected both from patient's circuit and cardioplegia circuit (1 L each). The samples, once concentrated (by centrifugation or, preferably, by filtration) should be decontaminated, using either the standard NALC- $2 \% \mathrm{NaOH}$ method, or cetylpyridinium chloride $0.005 \%$, before inoculation onto solid and liquid media for mycobacteria. Home-made methods for molecular detection of M. chimaera have also been developed $(3,57)$.

\section{Measures to mitigate transmission risk}

Having understood that HCU is involved in M. chimaera outbreaks, a number of risk mitigation measures have been implemented in hospitals. The most widely adopted include: replacement of $3 \mathrm{~T}$ HCUs produced before September 2014 with new ones and displacement of HCU outside the operating room (7). When impossible, it should be located distant from the operating table with the air exhaust as closer as possible to the room air suction exhaust.

HCU maintenance and decontamination must comply with the instructions for use in force since September $2014(9,12,33,52)$. 
Replacement of HCU internal tubing with evidence of biofilm is recommended and the water in the tank should be changed weekly using filtered water (18).

A written procedure should be implemented recording which individual HCU has been used in each intervention, to make possible, in case of later infection, to trace the device involved (31).

Not always such measures allowed eradication of $M$. chimaera (37); however, they could prevent new cases (54).

The hospital staff should be aware that, although the cases of M. chimaera outbreak were all linked to HCUs of a single brand, every non-airtight device with water reservoir can potentially become a vehicle of infections when used within operating rooms $(43,51,54)$

\section{Retrospective cases detection}

Due to different signs and symptoms of $M$. chimaera infection, a large number of health specialists (infectious diseases, cardiology, rheumatology, ophthalmology, haematology, pulmonology), together with primary care providers, have been alerted worldwide by national health authorities to maintain high level of attention for patients with a history of surgery with cardiopulmonary bypass and presenting with deep infections of unknown origin or with granulomatous disease sarcoidosis like $(16,17,28)$. Noteworthy is that a misdiagnosis of sarcoidosis often leads to start steroid treatment, thus causing a worsening of the disease $(10,15)$.

A measure implemented in many countries consists in contacting the subjects meeting the exposition criteria (open heart surgery with use of a 3 T HCU manufactured before September 2014) making them aware of risks and instructing them on how to recognize compatible signs and symptoms (38).

As for the 10 countries with the higher burden of open-heart surgery, 156-282 new cases/year have been recently estimated (42).

A national survey reported seven cases of $M$. chimaera infection following aortic or valvular surgery performed in Italy in 2017. The risk of infection was calculated 0.4-1 every 1000 interventions; median latency was two years, while mortality for patients requiring a new surgery was $50 \%$ (11).

Even in the best scenario of a prompt implementation of the risk mitigation measures in early 2016, several new cases are expected to emerge in the next two years.

\section{References}

1. A.T.S. Diagnosis and treatment of disease caused by nontuberculous mycobacteria. Am J Respir Crit Care Med 1997;2:S1-S25.

2. Achermann Y, Rossle M, Hoffmann M, et al. Prosthetic valve endocarditis and bloodstream infection due to Mycobacterium chimaera. J Clin Microbiol 2013;6:1769-73.

3. Acosta F, Perez-Lago L, Ruiz Serrano MJ, et al. Fast update of undetected Mycobacterium chimaera infections to reveal unsuspected cases. J Hosp Infect 2018;4:451-5.

4. Allen KB, Yuh DD, Schwartz SB, et al. Nontuberculous mycobacterium infections associated with heater-cooler devices. Ann Thorac Surg 2017;4:1237-42.

5. Asadi T, Mullin K, Roselli E, et al. Disseminated Mycobacterium chimaera infection associated with heatercooler units after aortic valve surgery without endocarditis. J Thorac Cardiovasc Surg 2018;6:2369-74.

6. Balsam LB, Louie E, Hill F, et al. Mycobacterium chimaera left ventricular assist device infections. J Card Surg 2017;6:402-4.

7. Barker TA, Dandekar U, Fraser N, et al. Minimising the risk of Mycobacterium chimaera infection during cardiopulmonary bypass by the removal of heater-cooler units from the operating room. Perfusion 2018;4:264-9.

8. Boyle DP, Zembower TR, Reddy S, et al. Comparison of clinical features, virulence, and relapse among Mycobacterium avium complex species. Am J Respir Crit Care Med 2015;11:1310-7.

9. C.D.C. Non-tuberculous mycobacterium (NTM) infections and heater-cooler devices interim practical guidance. 2016. Available from: http://www.cdc.gov/hai/pdfs/outbreaks/cdcnotice-heater-cooler-units-final-clean.pdf.

10. Cai Y, Landolfo K, Renew JR. Mycobacterium infection from a cardiopulmonary bypass heater-cooler unit in a patient with steroid-induced immunosuppression. Can J Anaesth 2017;5: 513-6.

11. Cappabianca G, Paparella D, D'Onofrio A, et al. Mycobacterium chimaera infections following cardiac surgery in Italy: results from a national survey endorsed by the Italian Society of Cardiac Surgery. J Cardiovasc Med (Hagerstown) 2018;12:748-55.

12. Chan T, Ling ML, Teng SY, et al. Microbiological monitoring of heater-cooler unit to keep free of Mycobacterium chimaera infection. Perfusion 2018;267659118787152.

13. Chand M, Lamagni T, Kranzer K, et al. Insidious risk of severe Mycobacterium chimaera infection in cardiac surgery patients. Clin Infect Dis 2017;3:335-42.

14. Cheng VCC, Wong SC, Chen JHK, et al. Mycobacterium chimaera-contaminated heater-cooler devices: the inner surface as the missing link? J Hosp Infect 2018;3:e157-8.

15. Chiesi S, Piacentini D, Salerno ND, et al. Disseminated Mycobacterium chimaera infection after open heart surgery in an Italian woman: a case report and a review of the literature. Infez Med 2017;3:267-9.

16. E.C.D.C. EU protocol for case detection, laboratory diagnosis and environmental testing of Mycobacterium chimaera infections potentially associated with heater-cooler units: case definition and environmental testing methodology. 2015. Available from: https://ecdc.europa.eu/sites/portal/files/media/en/publications/Publications/EU-protocol-for-M-chimaera.pdf.

17. E.C.D.C. Invasive cardiovascular infection by Mycobacterium chimaera associated with $3 \mathrm{~T}$ heater-cooler system used during open-heart surgery. 2016. Available from: https://ecdc.europa. eu/sites/portal/files/media/en/publications/Publications/RRAmycobacterium-chimaera-November-2016.pdf.

18. Garvey MI, Ashford R, Bradley CW, et al. Decontamination of heater-cooler units associated with contamination by atypical mycobacteria. J Hosp Infect 2016;3:229-34.

19. Gotting T, Klassen S, Jonas D, et al. Heater-cooler units: contamination of crucial devices in cardiothoracic surgery. J Hosp Infect 2016;3:223-8.

20. Griffith DE, Aksamit T, Brown-Elliott BA, et al. An official ATS/IDSA statement: Diagnosis, treatment and prevention of nontuberculous mycobacterial disease. Am J Respir Crit Care Med 2007;367-416.

21. Haller S, Holler C, Jacobshagen A, et al. Contamination during production of heater-cooler units by Mycobacterium chimaera potential cause for invasive cardiovascular infections: results of an outbreak investigation in Germany, April 2015 to February 2016. Euro Surveill 2016;21(17).

22. Hamad R, Noly PE, Perrault LP, et al. Mycobacterium chimaera infection after cardiac surgery: first Canadian outbreak. Ann Thorac Surg 2017;1:e43-5. 
23. Hsu JL, Bhatnagar U, Stys M, et al. M. chimaera: a multi-headed pathogen. S D Med 2018;6:246-50.

24. Jaworski R, Naumiuk L, Paczkowski K, et al. Mycobacterium chimaera - a new threat for cardiac surgical patients? Kardiochir Torakochirurgia Pol 2017;1:22-6.

25. Kasperbauer SH, Daley CL. Mycobacterium chimaera infections related to the heater-cooler unit outbreak: A guide to diagnosis and management. Clin Infect Dis 2018 [Epub ahead of print]

26. Lecorche E, Haenn S, Mougari F, et al. Comparison of methods available for identification of Mycobacterium chimaera. Clin Microbiol Infect 2018;4:409-13.

27. Lyman MM, Grigg C, Kinsey CB, et al. Invasive montuberculous mycobacterial infections among cardiothoracic surgical patients exposed to heater-cooler devices. Emerg Infect Dis 2017;5:796-805.

28. Ninh A, Weiner M, Goldberg A. Healthcare-associated Mycobacterium chimaera infection subsequent to heater-cooler device exposure during cardiac surgery. J Cardiothorac Vasc Anesth 2017;5:1831-5.

29. O’Neil CR, Taylor G, Smith S, et al. Mycobacterium chimaera infection after aortic valve replacement presenting with aortic dissection and pseudoaneurysm. Open Forum Infect Dis 2018; 2:ofy018.

30. Oda G, Ryono R, Lucero-Obusan C, et al. Epidemiologic review of veterans health administration patients with isolation of nontuberculous mycobacteria after cardiopulmonary bypass procedures. Infect Control Hosp Epidemiol 2017;9:1103-6.

31. Ogunremi T, Taylor G, Johnston L, et al. Mycobacterium chimaera infections in post-operative patients exposed to heatercooler devices: An overview. Can Commun Dis Rep 2017;5: 107-13.

32. Overton K, Mennon V, Mothobi N, et al. Cluster of invasive Mycobacteria chimaera infections following cardiac surgery demonstrating novel clinical features and risks of aortic valve replacement. Intern Med J 2018;12:1514-20.

33. Perkins KM, Lawsin A, Hasan NA, et al. Notes from the field: Mycobacterium chimaera contamination of heater-cooler devices used in cardiac surgery - United States. MMWR Morb Mortal Wkly Rep 2016;40:1117-8.

34. Robinson JO, Coombs GW, Speers DJ, et al. Mycobacterium chimaera colonisation of heater-cooler units (HCU) in Western Australia, 2015: investigation of possible iatrogenic infection using whole genome sequencing. Euro Surveill 2016;46: pii:30396.

35. Rudikoff AG, Ganocy TK I, Kansagra K, et al. Thoracolumbar osteomyelitis secondary to systemic Mycobacterium chimaera infection status post aortic valve rerplacement. J Cardiothorac Vasc Anesth 2018 [Epub ahead of print].

36. Sax H, Bloemberg G, Hasse B, et al. Prolonged outbreak of Mycobacterium chimaera infection after open-cest heart surgery. Clin Infect Dis 2015;1:67-75.

37. Schreiber PW, Kuster SP, Hasse B, et al. Reemergence of Mycobacterium chimaera in heater-cooler units despite intensified cleaning and disinfection protocol. Emerg Infect Dis 2016;10:1830-3.

38. Schreiber PW, Sax H. Mycobacterium chimaera infections associated with heater-cooler units in cardiac surgery. Curr Opin Infect Dis 2017;4:388-94.

39. Schweickert B, Goldenberg O, Richter E, et al. Occurrence and clinical relevance of Mycobacterium chimaera sp. nov., Germany. Emerg Infect Dis 2008;9:1443-6.

40. Scriven JE, Scobie A, Verlander NQ, et al. Mycobacterium chimaera infection following cardiac surgery in the United
Kingdom: clinical features and outcome of the first 30 cases. Clin Microbiol Infect 2018;11:1164-70.

41. Shafizadeh N, Hale G, Bhatnagar J, et al. Mycobacterium chimaera hepatitis: A new disease entity. Am J Surg Pathol 2019;43:244-50.

42. Sommerstein R, Hasse B, Marschall J, et al. Global health estimate of invasive Mycobacterium chimaera infections associated with heater-cooler devices in cardiac surgery. Emerg Infect Dis 2018;3:576-8.

43. Sommerstein R, Ruegg C, Kohler P, et al. Transmission of Mycobacterium chimaera from heater-cooler units during cardiac surgery despite an ultraclean air ventilation system. Emerg Infect Dis 2016;6:1008-13.

44. Stewardson AJ, Stuart RL, Cheng AC, et al. Mycobacterium chimaera and cardiac surgery. Med J Aust 2017;3:132-5.

45. Struelens MJ, Plachouras D. Mycobacterium chimaera infections associated with heater-cooler units (HCU): closing another loophole in patient safety. Euro Surveill 2016;46:pii:30397.

46. Svensson E, Jensen ET, Rasmussen EM, et al. Mycobacterium chimaera in heater-cooler units in Denmark related to isolates from the United States and United Kingdom. Emerg Infect Dis 2017;3:507-9.

47. Tortoli E, Fedrizzi T, Meehan CJ, et al. The new phylogeny of the genus Mycobacterium: The old and the news. Infect Genet Evol 2017;19-25.

48. Tortoli E, Nanetti A, Piersimoni C, et al. Performance assessment of new multiplex probe assay for identification of mycobacteria. J Clin Microbiol 2001;3:1079-84.

49. Tortoli E, Pecorari M, Fabio G, et al. Commercial DNA-probes for mycobacteria incorrectly identify a number of less frequently encountered species. J Clin Microbiol 2010;1:307-10.

50. Tortoli E, Rindi L, Garcia MJ, et al. Proposal to elevate the genetic variant MAC-A, included in the Mycobacterium avium complex, to species rank as Mycobacterium chimaera sp. nov. Int J Syst Evol Microbiol 2004;Pt 4:1277-85.

51. Trudzinski FC, Schlotthauer U, Kamp A, et al. Clinical implications of Mycobacterium chimaera detection in thermoregulatory devices used for extracorporeal membrane oxygenation (ECMO), Germany, 2015 to 2016. Euro Surveill 2016;46: pii:30398.

52. U.S. Food and Drug Administration. Mycobacterium chimaera infections associated with Sorin Group Deutschland $\mathrm{GmbH}$ Stockert 3T heater-cooler system: FDA safety communication. 2016. Available from: http://www.fda.gov/MedicalDevices/ Safety/AlertsandNotices/ucm504213.htm.

53. van Ingen J, Kohl TA, Kranzer K, et al. Global outbreak of severe Mycobacterium chimaera disease after cardiac surgery: a molecular epidemiological study. Lancet Infect Dis 2017; 10:1033-41.

54. Walker J, Moore G, Collins S, et al. Microbiological problems and biofilms associated with Mycobacterium chimaera in heater-cooler units used for cardiopulmonary bypass. J Hosp Infect 2017;3:209-20.

55. Wallace RJ Jr., Iakhiaeva E, Williams MD, et al. Absence of Mycobacterium intracellulare and presence of Mycobacterium chimaera in household water and biofilm samples of patients in the United States with Mycobacterium avium complex respiratory disease. J Clin Microbiol 2013;6:1747-52.

56. Zhang X, Lin J, Feng Y, et al. Identification of Mycobacterium chimaera in heater-cooler units in China. Sci Rep 2018;1:7843.

57. Zozaya-Valdes E, Porter JL, Coventry J, et al. Target-specific assay for rapid and quantitative detection of Mycobacterium chimaera DNA. J Clin Microbiol 2017;6:1847-56. 